\title{
El proceso de enseñanza aprendizaje mediante el uso de realidad virtual.
}

\section{The teaching-learning process through the use of virtual reality.}

\author{
Efraín Velasteguí López. ${ }^{1}$
}

\section{Resumen.}

La realidad virtual ha sido ampliamente señalada como un desarrollo tecnológico importante que puede apoyar al proceso de enseñanza y aprendizaje en muchos países han establecidos programas para introducir esta tecnología en diferentes niveles de enseñanza, la educación exige la necesidad de articular diversos tipos de estrategias para que ofrezca posibilidades a todo los estudiantes, el software educativo lo que busca es que los estudiante puedan interactuar con la nueva tecnología de la realidad virtual además es una herramienta de la tecnologías para la educación, capaz de transformar contundente y positivamente el proceso de enseñanza, además para el desarrollo educativo esta relación entre realidad virtual y software aparece en un momento en la historia de software especialmente como par aun apoyo en la educación a distancia de la forma que el software educativo, la tecnología ha progresado más rápido que nuestra habilidad para siquiera imaginar que vamos hacer con ella, además un software educativo cuyas características estructurales y funcionales sirvan para el apoyo entre el proceso de aprendizaje para un alumno se evidencia cuando el estudiante opera directamente el software educativo pero este caso sea de vital importancia la acción sea dirigida por un docente, no cabe duda al momento de utilizar la realidad virtual plantea numerosos problemas uno de ellos es el espacio físico ya que no un alumno no puede moverse con mayor libertad y sin peligro especialmente si hay que realizar viajes virtuales, aparte $\mathrm{d}$ esos el precio de estos dispositivos son costosos ya que ciertos establecimientos nos cumplen con recursos monetarios, el tiempo y el avance de la tecnología hacen que la manera de concebir la educación cambien permitiendo el acceso de recursos digitales.

Palabras claves: realidad virtual , desarrollo tecnológico , desarrollo educativo, software educativo.

\footnotetext{
${ }^{1}$ Ciencia digital, Ambato, Ecuador, luisefrainvelastegui@ cienciadigital.org
} 


\section{Abstract.}

Virtual reality has been widely noted as an important technological development that can support the teaching and learning process in many countries have established programs to introduce this technology in different levels of education, education requires the need to articulate various types of strategies so that offers possibilities to all students, educational software that seeks is that students can interact with the new technology of virtual reality is also a tool of technologies for education, capable of transforming forcefully and positively the teaching process, plus for educational development this relationship between virtual reality and software appears at a time in the history of software especially as even support in distance education in the way that educational software, technology has progressed faster than our ability to even imagine what are we doing In addition, an educational software whose structural and functional characteristics serve to support the learning process for a student is evidenced when the student directly operates the educational software but this case is of vital importance the action is directed by a teacher, There is no doubt when using virtual reality poses many problems one of them is the physical space and not a student can not move with greater freedom and without danger especially if you have to make virtual trips, apart from those the price of these devices they are costly since certain establishments fulfill us with monetary resources, the time and the advance of technology make the way of conceiving education change allowing the access of digital resources.

Keywords: virtual reality, technological development, educational development, educational software.

\section{Introducción}

Según (Pascual, 2016) Podemos definir la realidad virtual como un entorno de escenas generada por un dispositivo informático. El usuario se sumerge dentro de este entorno por medio de unas gafas o cacos de realidad virtual. Disponen de unas lentes que amplían el ángulo de visión creada la sensación de estas dentro de la escena, además se genera una imagen en 3D, que aumenta la sensación de realismo.

Es importante decir, que no es una pantalla en los ojos la tecnología de la realidad virtual es que consigue que el cerebro crea un mundo virtual en la que tu cuerpo reaccione como si fuera en la vida real.

Que cuando hablamos de realidad virtual, consiste en la inmersión sensorial en un nuevo mundo, basado en entornos reales que ha sido generado de forma artificia y que podemos visualizar con unas gafas de realidad virtual el objetivo de esta tecnología es crear un mundo ficticio del que puede formar parte de lo incluso no real, con el paso de las tecnologías la realidad virtual supone unos de los cambios tecnológicos más importantes.

Según (Porto, 2013 y Meriano, 2018) la realidad virtual ha suscitado un intenso debate en la sociedad, llegando a analizarse con intereses tanto sus ventajas como sus desventajas a favor se le atribuyen pros como estos:

- Es de gran utilidad para forman a ciertos profesionales que si n tienen verse en peligro o en situaciones complicadas durante su etapa de estudiantes 
- Se convierte en una herramienta muy útil para quienes, por enfermedades, no pueden salir de sus casas, ya que les permite, desde los cuatros paredes en la que están, poder conocer ciudades o espacios de todo el mundo que les gustaría visitar.

Las gafas de realidad virtual nos ofrecen ver solo lo que hay en la pantalla tiene sensores que reconocen la movilidad de la cabeza, en cuando hagas movimiento dentro del mundo virtual en el que estés, además es recomendado tener unos buenos auriculares para tener una mejor experiencia. Nos permite interactuar en el mundo virtual con todos nuestros sentidos sin embargo tiene un gran costo en el mundo del mercado la mayoría de estos sistemas se centran en la vista y el oído, hay otros que lo hacen a la vez con el tacto con el avance de la tecnología a un futuro no lejano también será al olfato y al gusto a la vez, la realidad virtual a sido incorporada en diferentes hábitos de las persona tales como video Juegos, educación y en el trabajo.

Según (karol, 2015) el software educativo o instruccional es un tipo de programa diseñado especialmente para ser usado por profesores y estudiantes para apoyar el proceso de enseñanza aprendizaje. Este software está diseñado con el único propósito de facilitar la enseñanza y el aprendizaje

El software educativo puede ofrecer oportunidades verdaderamente ventajosas para los estudiantes es el abierto, que no se enfoca en la enseñanza tanto como el aprendizaje creativo, el software educativo abierto ofrece un entorno en el cual los alumnos puedes explotar a su gusto, además apoya al labor del profesor en el proceso de aprendizaje unas de sus características es que deben tener elementos metodológicos que permita tener un mejor rendimiento en el aprendizaje además estos software pueden tener diferentes materias de formas muy diversa que tiene que ser muy acogida por el usuario y ofrecer un entorno de trabajo confortable, la mayoría de estos programas informáticos son nacidos con la finalidad educativa.

Según (Salvador, 2014) también conocido como software instruccional, el software educativo se trata de programas de enseñanzas que se sirven de las plataformas digitales para usarlas apoyo tanto a profesores como alumnos en los procesos de enseñanza aprendizajes. Y aunque puedan parecer muy novedosos, sus inicios se remontan a los años 60-70.

El software educativo ha sido abordado por diferentes autores atribuyendo disimilutes en el tema mismo a pesar de que se disponen las potencialidades y su absoluto basamento en los principios de la enseñanza para vinculación del proceso de enseñanza, el desarrollo del software educativo ha sido dirigido a las enseñanzas dirigidas a los diferente usuario, ciertamente aplica una gran cantidad de mejoramiento para los alumnos con el avance al pasar los años estos software están siendo mas didácticos para tener una mejor experiencia conforme va creciendo estos sistemas satisfacen a que los alumnos como docente tengan una mejor experiencia al utilizar estos tipos de software.

La experiencia del aprendizaje con la realidad virtual y aumentada 
Según (Suarez, 2017) La realidad virtual existe desde los años ochenta como una tecnología que e ha mantenido en constante evolución y aunque siempre se le ha dado atributos relacionados al mundo de los videojuegos y del entretenimiento el renacimiento de estos gadgets ha migrado a otras aplicaciones como la educación, permitiendo integrar la tecnología en sala de clases.

La realidad virtual puede ser aplicada en cual quiere lugar ya se en el campo, se está aplicando esta tecnología como en el turismo, marketing y también en la educación de todo el mundo como la educación Gestor, en el turismo lo que da a conocer son los lugares más populares en el mundo para que la persona tenga una mejor visión del lugar en el que va visitar, en el marketing hace que la persona tenga una mejor experiencia de compra a cualquier consumidor de forma inmersiva, es decir recreando los locales comerciales para que el usuario pueda visitarlo y recorrerlo a la vez de una forma virtual, el uso de la realidad aumentada en la educación es crear experiencia extraordinaria con el usuario y la conexión emocional, además ayuda a transformar la información textual en formato digital que facilita la información y a la vez ahorra el tiempo.

\section{Realidad virtual: así trasformara el sistema de educativo}

Según (Nuñez, 2016) la realidad virtual no solo se utiliza en el ámbito del entretenimiento aplicaciones al cine y a los videojuegos sino que también revolucionar a las aulas. Los expertos en pedagogía coinciden en que los beneficios de estos avances en el aprendizaje serán suficientemente nosotros como parte huevo en el sistema educativo.

Entre tantas opciones y nuevas tecnologías, la realidad virtual se posiciona como las herramientas ideales para crear nuevos ambientes educativos y es por el motivo que muchas escuelas han empezado a interesarse por esta herramienta de realidad virtual, la realidad virtual abre un nuevo mundo en el cual uno como estudiante no pueda moverse de su asiento, los contenidos de la realidad virtual son la clave para que un estudiante tenga su entorno educativo Sin embargo no debe deja pasar que un estudiante este en contacto con su entorno al momento de utilizar la realidad virtual

\section{Educación basada en experiencias}

Según (Aucas, 2017) la recreación de escenas de $360^{\circ}$ permite a los alumnos sentir los contenidos en lugar de simplemente leerlos o escucharlos en pocas palabras, estimula el proceso de aprendizaje, además las experiencias inmersiva consiguen alargar el tiempo de atención de los estudiantes y mejoras el trabajo en equipo.

Los procesos basados en competencias promueven habilidades relacionadas con la resolución de problemas, el aprendizaje autónomo y la capacidad para tomar decisiones además de auto dirigir sus acciones y analizar su impacto por lo que tienen un gran valor. Con el pasar del tiempo y el avance de la tecnología la educación va cambiando la manera de ser educada al momento de utilizar la realidad virtual que a la vez nos permite ingresar al acceso de estos recursos digitales tanto para los docente como a los estudiantes la realidad virtual va posesionando como una de las herramientas para la creación de nuevos ámbitos educativos. 


\section{Pero ¿Qué es esto de la realidad aumentada?}

Según (Moreno, 2014) la realidad aumentada es la tecnología que permite incorporar datos virtuales partir de un objeto del mundo real. Par ellos, necesitamos un dispositivo móvil, Tablet y etc., con una cámara, un software que lo procesa la información.

La realidad aumentada nos permite crear un mundo virtual mediante gráficos en $3 \mathrm{D}$ en lo cual es un mundo que no existe en la cual lo podemos ver, a la vez crear los que deseemos en dicho mundo de la realidad virtual. Cada vez las experiencias en este mundo son más reales en la cual es mundo de una gran fantasía ya que la realidad virtual es solo algo ficticio tiene una gran acogida en el ámbito de los videos juegos, también puede tener mundo de la medicina virtual.

La realidad aumentada es la que se le añade lo virtual, esto suena a realidad virtual, pero en realidad no lo es, la diferencia es que realidad virtual es así de lo real. Unas actividades de realidad aumentada es que puede ser usada en varios dispositivos con un sistema software que pueda aguantar todas las funciones de la realidad virtual

\section{¿Qué buscar en un software educativo?}

Según (Escobar, 2015) hay muchos tipos de software educativos y saber en qué consiste cada uno de ellos en invertir un poco de tiempo. Lo importante y lo que más debes tener en cuenta es que estos programas deben ser lo suficientemente flexibles para que pueda ser operado claro puedes asegurarte de que enseñe lo que realmente querías que aprendieran.

Un software educativo tiene que interactivo está destinado a apoyar a los alumnos a facilitar las diferentes actividades educativas a la ve resulta eficiente a los profesores en la preparación de las clases educativas que contribuye a que el estudiante y el profesor, en la docencia ayuda a que tenga beneficios pedagógicos, a los estudiantes estimula en el pensamiento que permite la retroalimentar lo aprendido.

Es muy importante en la educación por esta herramienta tecnológica permite simular las condiciones que existen en un aula o en un salón de clases, así el estudiante puede interactuar con el docente a través de videoconferencias, chat, correo electrónico y entre otros.

\section{Ventajas y Desventajas de software educativo}

Según (Acevedo, 2016) conociendo ahora lo que es un software educativo es indispensable saber las ventajas y desventajas.

Grafico N 1. Ventajas y desventajas del software educativo

\begin{tabular}{||l|ll|}
\hline \multicolumn{3}{|c|}{ Software Educativo } \\
\hline VENTAJAS & DESVENTAJAS \\
& & \\
\hline
\end{tabular}




\begin{tabular}{|c|c|}
\hline $\begin{array}{l}\text { - Se propician varios tipos de } \\
\text { aprendizajes que pueden ser grupales } \\
\text { o individuales. } \\
\text { - Favorece la construcción de } \\
\text { conocimientos y la reflexión por parte } \\
\text { del lector. } \\
\text { - Permite el acceso al conocimiento y a } \\
\text { la participación de las actividades. } \\
\text { - Incluye elementos para captar la } \\
\text { atención de los alumnos. } \\
\text { - Permite la participación en la } \\
\text { interacción y el desarrollo de nuevos } \\
\text { sistemas. }\end{array}$ & $\begin{array}{l}\text { - No hay un control o supervisión de } \\
\text { calidad d ellos contenidos. } \\
\text { - Debido a la facilidad de búsqueda } \\
\text { de información a través de este } \\
\text { medio, los alumnos pueden } \\
\text { utilizarlo como único recurso y } \\
\text { dejar de consultar otras fuentes. } \\
\text { - Algunos de los elementos } \\
\text { utilizados para captar la atención } \\
\text { de los alumnos como pueden } \\
\text { funcionar como distracciones. } \\
\text { - La herramienta wiki permite que } \\
\text { inescrupulosas, cambien la } \\
\text { información valiosa por } \\
\text { información inapropiada. }\end{array}$ \\
\hline
\end{tabular}

Fuente (Acevedo, 2016)

Elaborado: Jaqueline Ferrer Acevedo 2016.

Tipos de realidad Virtual

Grafico N 2. Tipos de realidad virtual

\begin{tabular}{|c|c|}
\hline \multicolumn{2}{|c|}{ Tipos de realidad Virtual } \\
\hline Virtual inmersiva & Virtual no inmersiva \\
\hline $\begin{array}{l}\text { Es decir, nos permite entrar en un } \\
\text { escenario como la rv, pero ya no eres un } \\
\text { simple espectador te conviertes en } \\
\text { protagonista puedes participar y decir que } \\
\text { quieres hacer o cómo quieres hacer esto se } \\
\text { consigue a través de los impulsos que tés } \\
\text { neuronas reciben y transmiten el cerebro } \\
\text { consideran un mayor realismo. Puedes } \\
\text { sentir sensaciones de todo tipo, ya que esa } \\
\text { inmersión en principio se debe conseguir a } \\
\text { través de la vista, oído, tacto, olfato y gusto. }\end{array}$ & $\begin{array}{l}\text { La realidad virtual no inmersiva utiliza } \\
\text { medios como el que actualmente nos ofrece } \\
\text { Internet en el cual podemos interactuar a } \\
\text { tiempo real con diferentes personas en } \\
\text { espacios y ambientes que en realidad no } \\
\text { existen sin la necesidad de dispositivos } \\
\text { adicionales a la computadora. La realidad } \\
\text { virtual no inmersiva ofrece un nuevo mundo } \\
\text { a través de una ventana de escritorio. }\end{array}$ \\
\hline
\end{tabular}

Fuentes (Picurelli, 2017 y Cerraraimd, 2008)

Elaborado: Tipos De Realidad Virtual 
La realidad virtual inmersiva Son aquella tecnología que posibilita al usuario, mediante el visor ver sumergirse en escenarios virtual como $360^{\circ}$ lo que propicia que el usuario se sienta confortable dentro de dichos escenarios.

La realidad virtual no inmersiva Nos ofrece un nuevo mundo a través de una ventana de escritorios. Este enfoque tiene varias ventanas sobre el inmersiva como el bajo costo la facilidad y la rapidez de aceptación de los usuarios.

\section{La realidad virtual como método de entretenimiento}

Según (Ortega, 2016) El ser humano siempre ha soñado con la realidad virtual, no solo en el aspecto del entretenimiento, sino también para aplicar esta tecnología en enfermedades cerebrales u otros tipos de avances técnicos. Un sueño que hemos visto muchas veces reflejado en diversos capos, en el cine.

Es algo innegable que la realidad virtual es una constante cada vez presente entre nosotros, una oportunidad de negocios que muchas empresas hace ya tiempo que están exprimiendo al máximo, como se puede ver que la realidad virtual impera en nuestro entorno tales como las redes sociales así como Facebook Y YouTube que permiten ver video en $360^{\circ}$, durante muchos años la realidad virtual trata de tomar liderazgo en el ámbito virtual pero con el pasar el tiempo esta tecnología ha mejorado dando a ofrecer un realismo para sus usuarios, un realismos en años anteriores no existía pero en estos momentos son elementos fundamentales para a la vez la realidad virtual busca que los usuarios interactúen en sus plataformas virtuales también hay muchas alternativas que se pueden acoplar a los usuarios de acuerdo a los gusto que el posee.

\section{¿Cómo funciona la realidad virtual?}

Según (Crotf, 2018) Tal y como hemos vistos ha habido muchas variedades de dispositivos y tecnologías a lo largo de los años, aun así, nos centramos en las más recientes todas comparten una seria de ingredientes imprescindibles para funcionar y conseguir sumergir al usuario en un nuevo mundo.

La realidad virtual ha sido desarrollada con la capacidad de emitir infinidad de reacciones antes la las acciones ejecutadas por los usuarios, brindando un marco que ofrezca una experiencia inmersa, lo que hace la realidad virtual es eliminar la frontera que siempre ha existido entre lo real y lo irreal, difuminando los límites que separan ambos mundo es impresionante como va avanzando la realidad virtual con el pasar de los años, la realidad virtual en su ámbito de campo de visión tiene que ser amplio para que el usuario tenga una mejor experiencia con el mundo ficticio, lo que hace la realidad virtual es conocer el tamaño de los objetos a la vez conocer las dimensiones en la que lo rodea la segunda es la movilidad en el usuario tenga en el mundo virtual todo estos se debe tener en cuenta al momento de crear gafas virtuales también las resolución de los gráficos tiene que ser la adecuad en todo momento tiene ser algo real para sentirse uno como usuario cómodamente

\section{Característica y objetivo de la realidad virtual}


Según (Box, 2017) avanzando la tecnología la realidad virtual ha tomado característica que simula casi a la perfección al mundo físico, a veces confundiendo a los jugadores.

- Se representa en un espacio 3d interactivo que responde a los gestos del usuario.

- Permite tener vivencias de la forma controlada, como escalar una montaña, practicar submarinismo o nadar entre tiburones.

- Opera tiempo real, al mismo tiempo que el dispositivo se mueve también lo hace el mundo virtual.

- Gracias a los estímulos que siente el usuario, permiten que la realidad virtual sea un poco más real.

- Necesita de unos aparatos muy específicos para poder funcionar y que, dependiendo del diseño puedan ser de coste muy elevado.

La realidad virtual se caracteriza por una serie de aspectos, mediante esas especificaciones técnicas que han sido desarrolladas, modificadas y mejoradas con los años hoy es posible acércanos a lo que es real que nos brindan a conocer algo nuevo a la vez, lo que hace es crear mundo virtuales hoy en día existen diferentes aplicaciones en la cual te ofrecen este entorno virtual lo que se pretende es generar un entorno virtual que no pueda ser diferenciado de lo realidad normal, ya que la realidad virtual lo que hace es sustituir el mundo real por algo virtual ha sido siempre lograr una creación de un mundo irreal pero a la vez posible.

Uso de la realidad virtual, en la educación del futuro en centros educativos del Ecuador

Según (Unesco 2013) los datos estadísticos de Unesco en ecuador, el 18\% de los establecimientos educativos primarios cuentan con acceso a internet y un $60 \%$ los establecimientos secundarios, estos datos fueron publicados hasta el año 2010.

Grafico N 3: Gráfico de acceso a internet en establecimientos escolares del Ecuador

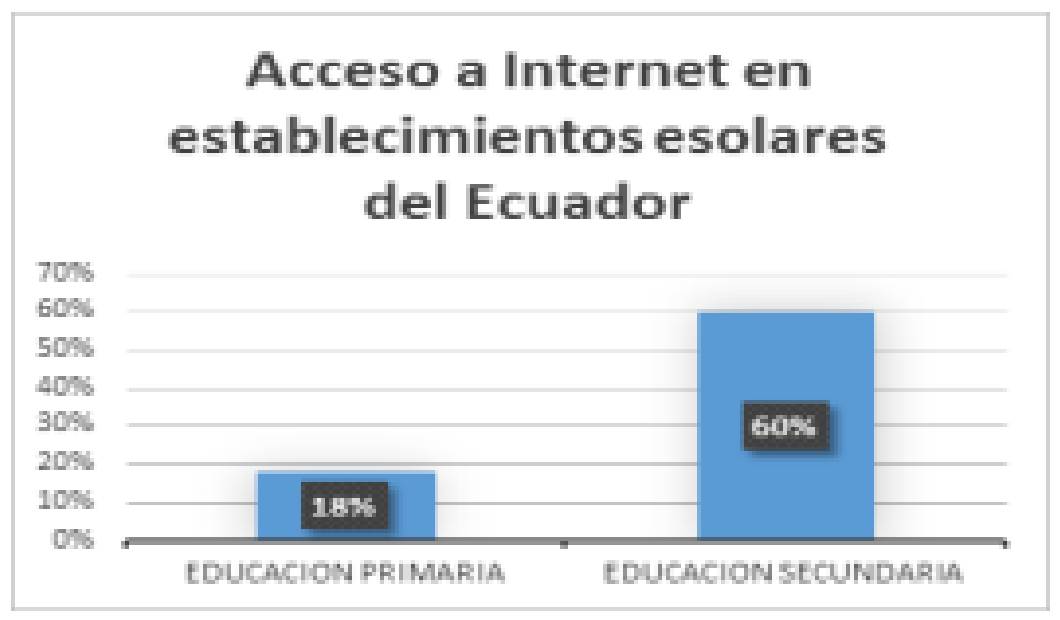

Fuente: (Unesco 2013)

Elaborado: Liliana Ivett Urquiza Mendoza. 
Nos menciona la Unesco es que, si en el ecuador se está implementando la realidad virtual como un medio de enseñanza, la realidad virtual podría ser integrada si los docentes fueran capacitados en el manejo y uso de esta nueva tecnología a la vez portaría con la educación, pero sería necesario la participación de las entidades del gobierno encargaba en el sistema de educación de Ecuador.

\section{Conclusiones}

$>$ La realidad virtual se refiere al uso de las computadoras y otros elementos coordinados por ella, para la simulación dinámica y tridimensional con alto contenido grafico acústico y táctil que logra simular un mundo semi real.

$>$ El desarrollo de ambientes virtuales adecuados para el uso de la educación constituye un área muy poco explorados, pero a la vez existe simuladores de programas de entreteniendo.

\section{Referencias Bibliográficas}

Pascual, j. (2018). Realidad Virtual: 25 preguntas y respuestas que debes conocer. [online] computerhoy. Available at: https://computerhoy.com/noticias/zonagaming/realidad-virtual-25-preguntas-respuestas-que-debes-conocer-42543 [Accessed 28 Oct. 2018].

Pérez Porto, J. y Merino, M. (2013). realidad virtual. [online] definicion. Available at: https://definicion.de/realidad-virtual/ [Accessed 30 Oct. 2018].

karol (2015). Software Educativo: Características, Tipos, Ventajas, Desventajas - Lifeder. [online] Lifeder. Available at: https://www.lifeder.com/software-educativo/ [Accessed 30 Oct. 2018].

Salvador, I. (2014). Software educativo: tipos, características y usos. [online] Psicologiaymente.com. Available at: https://psicologiaymente.com/desarrollo/software-educativo [Accessed 30 Oct. 2018].

Suarez, I. (2017). la experiencia del aprendizaje con la realidad virtual y aumentada. [Blog] u-planner. Available at: https://www.u-planner.com/es/blog/lasaplicaciones-de-la-realidad-virtual-en-la-educacion-superior [Accessed 30 Oct. 2018].

Nuñez, P. (2016). Realidad virtual: así transformará el sistema educativo. [Blog] elmundo. Available at: https://www.elmundo.es/andalucia/2016/04/28/57223250ca47418b128b4651.h tml [Accessed 30 Oct. 2018].

Aucas (2017). la realidad virtual y su aplicación en el aula. [Blog] auca.es. Available at: : https://www.auca.es/la-realidad-virtual-aplicacion-aula/ [Accessed 30 Oct. 2018]. 
Moreno, M. (2014). pero, ¿qué es esto de la realidad aumentada?. [Blog] nubemia. Available at: https://www.nubemia.com/realidad-aumentada-en-la-educacion/ [Accessed 30 Oct. 2018].

Escobar, R. (2015). Ventajas y desventajas de utilizar un software educativo. [Blog] Sweetter. Available at: http://sweetter.net/ventajas-y-desventajas-de-utilizar-unsoftware-educativo/ [Accessed 30 Oct. 2018].

ACEVEDO, J. (2016). ventajas y desventajas. [online] software educativo. Available at: https://jaqueferrer.weebly.com/ventajas-y-desventajas.html [Accessed 4 Nov. 2018].

Picurelli, L. (2017). Realidad virtual inmersiva ¿Qué es y cómo aplicarla a las apps?. [Blog] yeeply. Available at: https:/www.yeeply.com/blog/realidad-virtualinmersiva-que-es-y-como-aplicarla-apps/ [Accessed 30 Oct. 2018].

Cerraraimd, F. (2008). Realidad Virtual Inmersiva y No Inmersiva. [Blog] felipecarraraimd. Available at: http://felipecarraraimd.blogspot.com/2008/09/realidad-virtual-inmersiva-yno.html [Accessed 30 Oct. 2018].

Ortega, J. (2016). un repaso a la historia de la realidad virtual. [Blog] es.ign. Available at: https://es.ign.com/realidad-virtual/109691/feature/un-repaso-a-la-historiade-la-realidad-virtual [Accessed 30 Oct. 2018].

Croft, P. (2018). Realidad Virtual: origen, actualidad y futuro. [Blog] as. Available at: https://as.com/meristation/2018/01/10/reportajes/1515567480_172151.html [Accessed 30 Oct. 2018].

Crosft, p. (2018). Realidad Virtual: origen, actualidad y futuro. [Blog] as. Available at: https://as.com/meristation/2018/01/10/reportajes/1515567480_172151.html [Accessed 4 Nov. 2018].

Box, V. (2017). Qué características posee la realidad virtual. [Blog] vr-box.games. Available at: https://vr-box.games/caracteristicas-de-la-realidad-virtual/ [Accessed 28 Oct. 2018].

UNESCO (2013). Uso de TIC en Educacion en Am ' erica ' Latina y el Caribe. Analisis regional de la integraci ' on de las ' TIC en la educacion y de la aptitud digital ( e - readiness ').

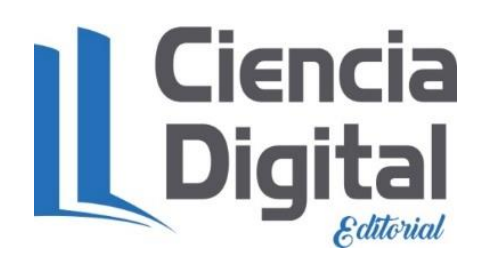




\section{Para citar el artículo indexado.}

Velasteguí E. (2018). El proceso de enseñanza aprendizaje mediante el uso de realidad virtual. Revista electrónica Explorador Digital 2(2), 5-15. Recuperado desde:

http://cienciadigital.org/revistacienciadigital2/index.php/exploradordigital/article/view/328/7 $\underline{40}$

\section{Ciencia \\ Digital \\ Editorial}

El artículo que se publica es de exclusiva responsabilidad de los autores y no necesariamente reflejan el pensamiento de la Revista Explorador Digital.

El articulo queda en propiedad de la revista y, por tanto, su publicación parcial y/o total en otro medio tiene que ser autorizado por el director o editor de la Revista Explorador Digital.
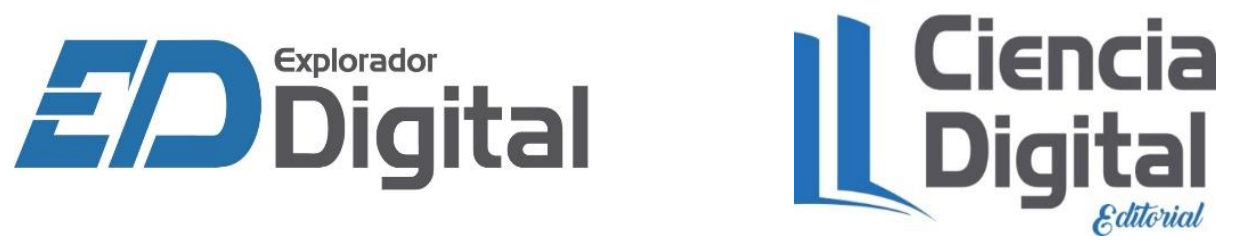